unanimously accepted. The work aims to specify the pathological and clinical features and to highlight the prognostic factors of these tumors.

Methodology Our study was retrospective and descriptive including 49 cases of primary borderline mucinous tumors of the ovary, diagnosed at the Department of Anatomical Pathology and Cytology of Salah Azaiez Institute, for a period of 27 years, going from 1992 to 2019.

Result(s)* The mean age of our patients was 48 years old. Histologically, the cases were divided into 34 cases of pure MBT, 13 cases with intraepithelial carcinoma, and 2 cases associating an intraepithelial carcinoma with microinvasion. The majority of our cases were classified FIGO I and only one case was FIGO III. 14 patients received conservative treatment and 32 received radical treatment. The treatment wasn't specified in 3 patients. The progress was good in the majority of cases. Only one patient had a contralateral recurrence after a follow-up period of 3 years. There was no significant difference regarding the risk of recurrence and risk factors such as age, gestation, hormonal contraception, hormonal status, FIGO stage, presence of peritoneal pseudomyxoma, intraepithelial carcinoma, and microinvasion.

Conclusion* The prognosis of TMBL depends closely on their FIGO stage, stage I tumors have a good prognosis. The presence of intraepithelial carcinoma does not influence their prognosis. However, it is necessary to multiply samples to avoid missing a carcinomatous focus with an anarchic invasion of the stroma which constitutes a poor prognosis factor.

\section{OUTSTANDING CLINICAL DILEMMA OF RAISED ALPHA- FETO PROTEIN WITHOUT HISTOLOGICAL, RADIOLOGICAL AND SURGICAL CONFIRMATION OF ORIGIN}

${ }^{1} \mathrm{M}$ Chakrabarti, ${ }^{2} \mathrm{C}$ Goswami, ${ }^{3} \mathrm{~S}$ Roy. ${ }^{1}$ Columbia Asia Hospital Salt Lake, Kolkata, Gynaecological Oncology, Kolkata, India; ${ }^{2}$ AMRI Hospital - Dhakuria, Medical Oncology, Kolkata, India; ${ }^{3}$ Drs. Tribedi and Roy Diagnostic Laboratory, Pathology, Kolkata, India

\subsection{6/ijgc-2021-ESG0.499}

Introduction/Background* Elevated Alpha-Feto Protein (AFP) in a young female with ovarian mass is virtually diagnostic of Malignant Germ Cell Tumours ${ }^{1}$. We describe a case with outstanding clinical dilemma where the cause of raised AFP remains unsubstantiated.

Methodology A 13 year old girl presented with lower abdominal discomfort. Ultrasound evaluation suggested large left adnexal dermoid cyst. AFP was elevated at $728 \mathrm{ng} / \mathrm{dL}^{2}$. CT scan showed left adnexal mass and a suspicious small lesion in liver without any other abdominal lesion. She was overweight with grade-2 fatty liver, mildly raised alkaline-phosphatase, hepatomegaly with family history of liver malignancy.

A torted left-adnexal smooth mass was removed during surgery. Peritoneal washing, opposite ovary and systematic peritoneal cavity examination were unremarkable. HPE was inconclusive as the tumour was necrotic. After a gap she attended for follow up and on $4^{\text {th }}$ postoperative-month AFP level was $534.84 \mathrm{ng} / \mathrm{dL}$.

Further CT and MRI did not reveal any liver lesions. Right ovary had features of polycystic ovary (PCO). On $5^{\text {th }}$ postoperative-month PET/CT revealed FDG avid $3.5 \mathrm{~cm}$ solid-cystic lesion in right adnexa with SUV Max of 5.6, suspicious of malignancy. Patient and family underwent thorough counselling between extent of surgeries vs chemotherapy.
Result(s)* On second surgical evaluation the right ovary appeared normal and wedge biopsy was benign. Soon after surgery she attained menarche. At $19^{\text {th }}$ month post index surgery, AFP remained elevated; steady at mid-500 ng/dL level without radiological abnormalities. She is on pathway for weight reduction and regular follow up.

Conclusion* This case report enriches the limited literature on rare reasons for non-hepatic and non-germ cell tumour AFP elevation. Moderate metabolic avidity on PET/CT may signify intense hormonal activities in premenstrual ovary ${ }^{3}$. Causes like Hereditary Persistence of AFP (HPAFP), persistent elevated AFP due to non-hereditary mutations in enhancer and silencer regions of AFP transcription ${ }^{4}$, dietary inflammatory agents and autoimmune neuroinflammation ${ }^{5}$ are some of issues which need further research. It is important to recognise these conditions to avoid inappropriate clinical decisions and minimise anxiety level of all concerned ${ }^{4}$. There is need for worldwide registry and in-depth research with genome and exome sequencing to explore raised AFP with unaccommodating classical pathologies.

\section{IMPACT OF LYMPHADENECTOMY ON SURVIVAL OF PATIENTS WITH SEROUS ADVANCED OVARIAN CANCER AFTER NEOADJUVANT CHEMOTHERAPY: A NATIONAL MULTICENTER STUDY}

${ }^{1} V$ Bund*, 'L Lecointre, ${ }^{2} \mathrm{M}$ Velten, ${ }^{3} \mathrm{~L}$ Ouldamer, ${ }^{4} \mathrm{~S}$ Bendifallah, ${ }^{5} \mathrm{M}$ Koskas, ${ }^{6} \mathrm{PA}$ Bolze, ${ }^{7} \mathrm{P}$ Collinet, ${ }^{8} \mathrm{G}$ Canlorbe, ${ }^{9} \mathrm{~T}$ Touboul, ${ }^{10} \mathrm{C}$ Huchon, ${ }^{11} \mathrm{C}$ Coutant, ${ }^{1} \mathrm{E}$ Faller, ${ }^{1} \mathrm{~T}$ Boisramé, 12. Gantzer, ${ }^{13} \mathrm{M}$ Demarchi, ${ }^{1} \mathrm{JJ}$ Baldauf, ${ }^{14} \mathrm{M}$ Ballester, ${ }^{15} \mathrm{~V}$ Lavoue, ${ }^{1} \mathrm{C}$ Akladios. ${ }^{1}$ Department of Gynecologic Surgery, Hôpitaux Universitaires de Strasbourg, Strasbourg, France; ${ }^{2}$ Department of Public Health, Hôpitaux Universitaires de Strasbourg, Strasbourg, France; ${ }^{3}$ Department of Gynecology, Hôpital Universitaire de Tours, Tours, France; ${ }^{4}$ Department of Gynaecology and Obstetrics, Hôpital Tenon, AP-HP, Paris, France; ${ }^{5}$ Department of Gynecology, Hôpital Bichat, AP-HP, Paris, France; ${ }^{6}$ Gynecological Surgery Service, CHU Lyon-Sud, Pierre-Bénite, Lyon, France; ' Department of Gynecological Surgery, Hôpital Jeanne De Flandre, CHRU, Lille, France; ${ }^{8}$ Department of Gynecologic and Breast Surgery and Oncology, Hôpital la Pitié Salpétrière, AP-HP, Paris, France; ${ }^{9}$ Department of Obstetrics and Gynaecology, Centre Hospitalier Intercommunal, Créteil, France; ${ }^{10}$ Department of Gynecology, Centre Hospitalier de Poissy, Poissy, France; ${ }^{11}$ Department of Surgical Oncology, Georges-Francois Leclerc Cancer Center, Dijon, France; ${ }^{12}$ Department of Medical Oncology, Hôpitaux Universitaires de Strasbourg, Strasbourg, , France; ${ }^{13}$ Medical Oncology Department, Centre Paul Strauss, Strasbourg, France; ${ }^{14}$ Department of Gynecologic and Breast Surgery, Groupe Hospitalier Diaconesses Croix, Saint-Simon, Paris, France; ${ }^{15}$ Department of Gynecologic Surgery, Hôpital Universitaire de Rennes, Rennes, France

\subsection{6/ijgc-2021-ESG0.500}

Title: Impact of Lymphadenectomy on Survival of Patients with Serous Advanced Ovarian Cancer After Neoadjuvant Chemotherapy: A French National Multicenter Study (FRANCOGYN).

Introduction/Background* The population of interest to this study comprised individuals with advanced-stage ovarian carcinoma who were exposed to neoadjuvant chemotherapy (NAC) followed by interval debulking surgery (IDS). Those who had not received systematic lymphadenectomy (SL; Group 1) were compared to those who had received SL (Group 2). Outcome measures included progression-free survival (PFS), overall survival (OS), and surgical complications. (Group 1).

Methodology This was a retrospective, multicenter cohort study in nine referral centers of France between January 2000 and June 2017. OS analysis using the multivariate Cox regression model was performed. PFS and surgery-related morbidity were analyzed. 